\title{
Dissecting the 3D structure of elliptical galaxies with gravitational lensing and stellar kinematics
}

\author{
Matteo Barnabè $\grave{1}^{1,2}$, Chiara Spiniello ${ }^{3}$ and Léon V. E. Koopmans ${ }^{4}$ \\ ${ }^{1}$ Dark Cosmology Centre, Niels Bohr Institute, University of Copenhagen, \\ Juliane Maries Vej 30, 2100 Copenhagen Ø, Denmark \\ email: mbarnabe@dark-cosmology.dk \\ ${ }^{2}$ Niels Bohr International Academy, Niels Bohr Institute, University of Copenhagen, \\ Blegdamsvej 17, 2100 Copenhagen Ø, Denmark \\ ${ }^{3}$ Max Planck Institute for Astrophysics, Karl-Schwarzschild-Strasse 1, \\ 85740 Garching, Germany \\ ${ }^{4}$ Kapteyn Astronomical Institute, University of Groningen, P. O. Box 800, \\ 9700 AV Groningen, The Netherlands
}

\begin{abstract}
The combination of strong gravitational lensing and stellar kinematics provides a powerful and robust method to investigate the mass and dynamical structure of early-type galaxies. We demonstrate this approach by analysing two massive ellipticals from the XLENS Survey for which both high-resolution HST imaging and X-Shooter spectroscopic observations are available. We adopt a flexible axisymmetric two-component mass model for the lens galaxies, consisting of a generalised NFW dark halo and a realistic self-gravitating stellar mass distribution. For both systems, we put constraints on the dark halo inner structure and flattening, and we find that they are dominated by the luminous component within one effective radius. By comparing the tight inferences on the stellar mass from the combined lensing and dynamics analysis with the values obtained from stellar population studies, we conclude that both galaxies are characterised by a Salpeter-like stellar initial mass function.
\end{abstract}

Keywords. galaxies: elliptical and lenticular, cD - galaxies: structure - galaxies: kinematics and dynamics - gravitational lensing: strong

\section{Introduction}

Understanding the formation and evolution mechanisms of early-type galaxies (ETGs) remains a crucial challenge in present-day astrophysics. In recent years, high-resolution simulations of galaxy evolution, including both baryons and dark matter (e.g. Genel et al. 2014), have progressed enough that it is finally becoming possible to investigate in detail the physical properties of simulated systems and compare them to the corresponding properties of observed ellipticals in the local Universe. Whereas nearby ETGs have been the object of intense scrutiny, with stellar dynamics being the most often employed diagnostic tool (see e.g. Thomas et al. 2011; Cappellari et al. 2013), little is known about the detailed structure of ellipticals beyond redshift $z \approx 0.1$, despite the obvious importance of providing observational constraints to simulations throughout cosmic history.

At higher redshift, however, massive galaxies occasionally act as strong gravitational lenses: for such systems it becomes possible to supplement stellar kinematics with the lensing constraints to investigate the mass structure of these distant galaxies to a level that is comparable with what can be obtained for nearby objects (see e.g. Czoske et al. 2008; Koopmans et al. 2009; van de Ven et al. 2010; Barnabè et al. 2010, 2011). High quality combined data-sets, including extended kinematics, make it possible to 
disentangle the contributions of dark and luminous matter to the total mass budget, which in turns allows one to draw inferences also on the stellar initial mass function (IMF) of the system, by comparing the results with the stellar masses obtained from independent stellar population synthesis (SPS) analysis assuming a variety of IMF profiles. We demonstrate the power of this approach by applying a self-consistent combined lensing and dynamics analysis to two massive ETGs from the XLENS Survey (Spiniello et al. 2011) for which high signal-to-noise X-Shooter spectroscopic observations are at hand.

\section{Observations}

Both massive ellipticals SDSS J0936+0913 (velocity dispersion $\sigma \simeq 245 \mathrm{kms}^{-1}$; redshift $z=0.164)$ and SDSS J0912+0029 $\left(\sigma \simeq 325 \mathrm{kms}^{-1} ; z=0.190\right)$ were first observed as part of the SLACS Survey (Bolton et al. 2008). The high-resolution images needed for the lensing modelling were obtained with $H S T$ ACS through the $F 814 W$ filter. Elliptical B-spline models of the lens galaxies were subtracted off the images to isolate the structure of the gravitationally lensed background sources.

High signal-to-noise $(\mathrm{S} / \mathrm{N}>50) \quad U V B-V I S$ X-Shooter spectra are available for both systems, and were used both to carry out SPS analysis and derive long-slit spatially resolved stellar kinematics up to about one effective radius, $R_{\mathrm{e}}$.

\section{Modelling}

A joint self-consistent modelling of all the available lensing and kinematic constraints to derive inferences on the mass and dynamical structure of the two systems is conducted with the fully Bayesian CAULDron code (Barnabè \& Koopmans 2007; Barnabè et al. 2012). The lens galaxy is described by a flexible and realistic two-component axisymmetric mass model consisting of (i) a generalised Navarro-Frenk-White (gNFW) halo characterised by four free parameters (the inner density slope $\gamma$, the axial ratio $q_{\mathrm{h}}$, the halo concentration parameter $c_{-2}$ and the virial velocity $v_{\text {vir }}$ ) and (ii) a mass profile for the baryonic component obtained by de-projecting the multi-Gaussian expansion fit (MGE) to the observed surface brightness distribution of the galaxy, characterised by one free parameter (the total stellar mass $M_{\star}$ that sets the normalisation of the luminous distribution). The stellar kinematics is modelled with the Jeans anisotropic method (JAM) developed by Cappellari (2008), including the meridional plane orbital anisotropy $b=\sigma_{R}^{2} / \sigma_{z}^{2}$ as a free parameter.

\section{Results and discussion}

The full inferences on the model parameters obtained from the combined CAULDRON analysis are expressed as multivariate posterior probability distribution functions (PDFs), encapsulating all the information on the uncertainties in a statistically rigorous way. The $1 \mathrm{D}$ and 2D marginalised posterior PDFs for the individual parameters can be visualised as a familiar corner plot, as shown in Fig. 1 for galaxy J0912.

Both systems are found to be markedly dominated by the baryonic component within the inner $R_{\mathrm{e}}$, with a dark matter fraction $f_{\mathrm{DM}}=0.06_{-0.05}^{+0.10}$ for J0936 and $f_{\mathrm{DM}}=0.18_{-0.08}^{+0.08}$ for the more massive system J0912. The dark halo structure of both galaxies $\left(c_{-2}=\right.$ $6.3_{-4.1}^{+18.4}$ and $v_{\mathrm{vir}}=124_{-88}^{+160} \mathrm{kms}^{-1}$ for J0936; $c_{-2}=7.2_{-2.7}^{+3.8}$ and $v_{\mathrm{vir}}=470_{-130}^{+160} \mathrm{kms}^{-1}$ for J0912) is consistent with the concentration-virial velocity relation predicted from pure $N$-body simulations (Macciò et al. 2008): thus, within the fairly broad uncertainties, 


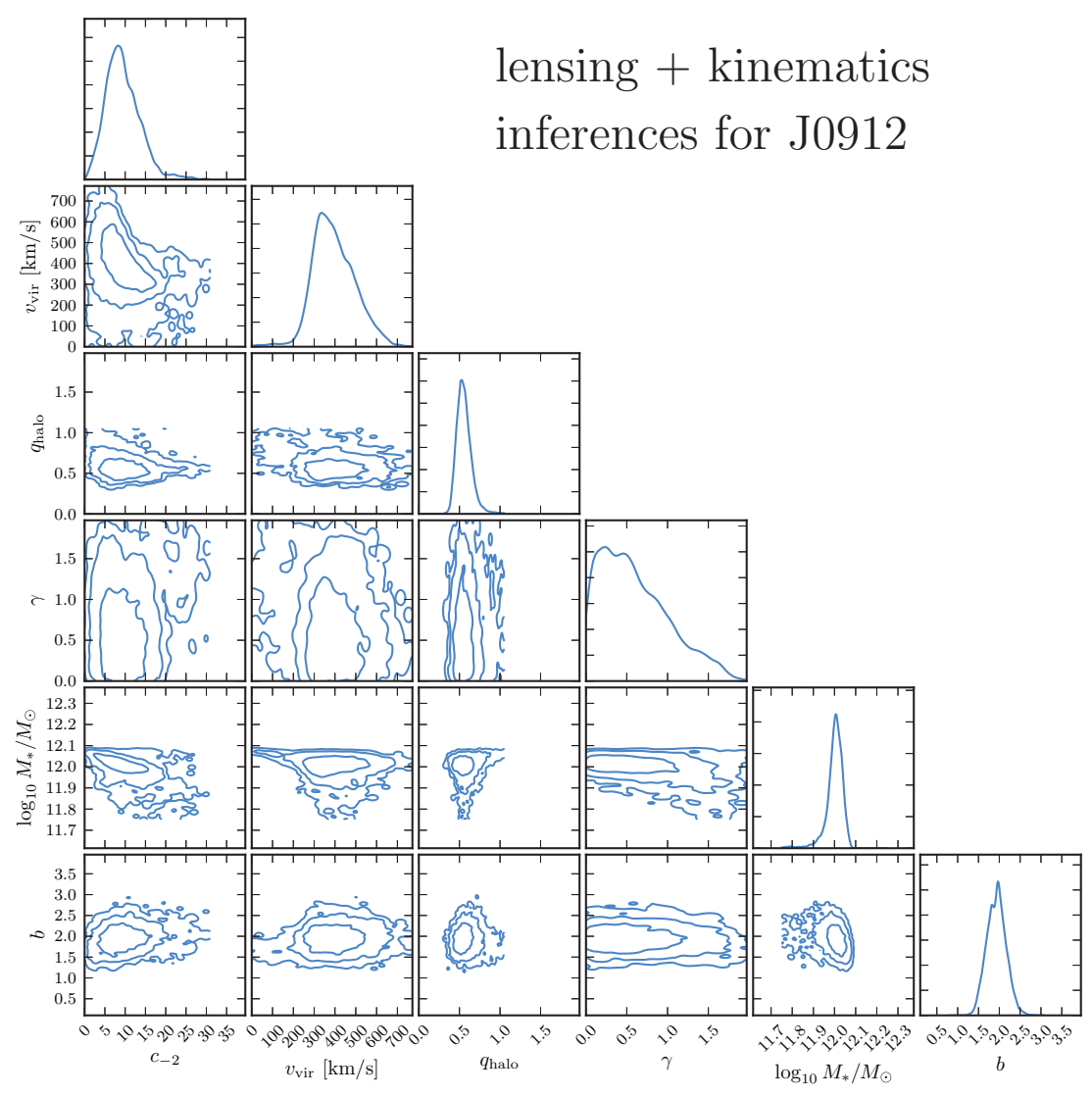

Figure 1. Marginalised $1 \mathrm{D}$ and $2 \mathrm{D}$ posterior PDFs for the six model parameters for galaxy J0912, using the combined constraints from lensing and kinematics. The three contours indicate the regions containing, respectively, $68 \%, 95 \%$ and $99.7 \%$ of the probability.

there is no obvious evidence for contraction or expansion of the dark halo in response to galaxy formation. The inner slope of the dark halo is basically unconstrained for J0936 $\left(\gamma=0.92_{-0.64}^{+0.72}\right)$, while for J0912 we can conclude that steep slopes are disfavoured $\left(\gamma=0.46_{-0.30}^{+0.41}\right)$. The two haloes differ in terms of flattening, with J0936 having a more spherical halo $\left(q_{\mathrm{h}}=0.93_{-0.18}^{+0.25}\right)$, while the halo of J0912 is clearly oblate $\left(q_{\mathrm{h}}=0.54_{-0.08}^{+0.10}\right)$.

The two lens galaxies have dissimilar dynamical structures within the probed region: J0936 $\left(b=0.89_{-0.31}^{+0.37}\right)$ is consistent with being a semi-isotropic system, while the velocity dispersion ellipsoid of J0912 is distinctly flattened $\left(b=1.94_{-0.21}^{+0.24}\right)$.

By comparing the stellar mass inferred from the combined lensing and dynamics modelling with the one derived from SPS analysis of X-Shooter spectra we can also put robust constraints on the normalisation of the stellar IMF, as illustrated in Fig. 2 for J0912. We determine that both galaxies are consistent with having a Salpeter IMF (i.e., slope $x=2.35$ for a power-law IMF profile $d N / d m \propto m^{-x}$ ), in agreement with the findings for local massive ETGs from both spectroscopic and dynamical studies (e.g. van Dokkum \& Conroy 2010; Cappellari et al. 2012). Moreover, we can rule out both a Chabrier and a "super-Salpeter" $(x \geqslant 3)$ IMF with a high degree of confidence. The XLENS data-set makes it possible also to determine the IMF in a completely independent way from a spectroscopic simple stellar population analysis of the optical line-strength indices, using the approach outlined by Spiniello et al. 2014: the IMF slopes obtained with this method 


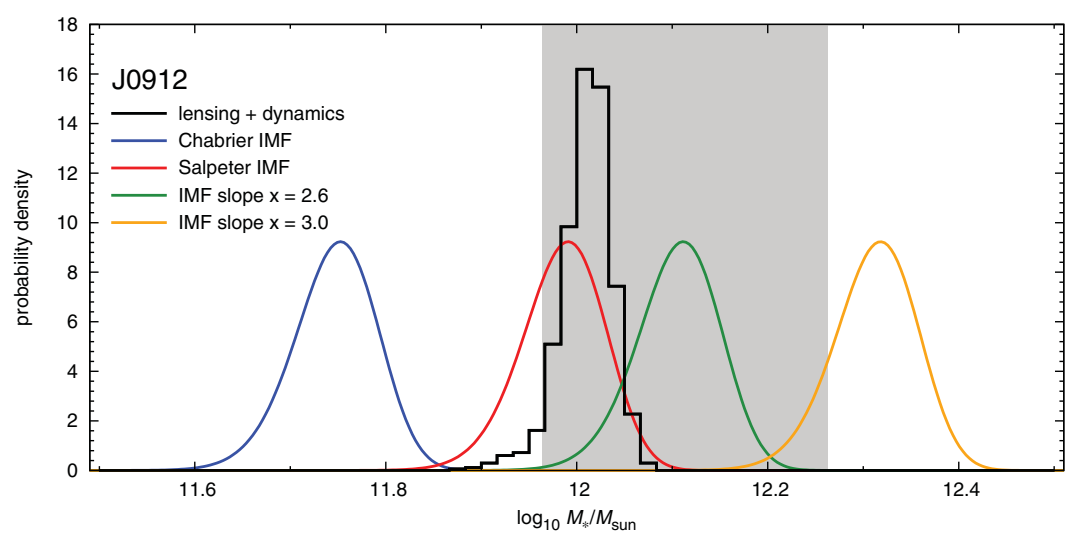

Figure 2. Comparison of the total stellar mass inferred for J0912 from the combined lensing and dynamics study (histogram) with $M_{\star}$ obtained from SPS analysis, for different values of the IMF slope (coloured curves). The green curve indicates $M_{\star}$ for the best-fit IMF slope determined from an independent method based on spectroscopic simple stellar population modelling of line-strength indices, with the grey band showing the 1-sigma uncertainties around that value.

$(x=2.10 \pm 0.15$ for J0936 and $x=2.60 \pm 0.30$ for J0912) are fully consistent with the much tighter lensing and dynamics results. Finally, the constraints from these two complementary approaches can be combined to investigate the elusive low-mass cut-off of the IMF, as demonstrated by Barnabè et al. (2013). Work is ongoing to extend this analysis to the entire XLENS sample of 10 massive lens ETGs.

\section{Acknowledgments}

The Dark Cosmology Centre is funded by the Danish National Research Foundation.

\section{References}

Barnabè, M., Auger, M. W., Treu, T., et al. 2010, MNRAS, 406, 2339

Barnabè, M., Czoske, O., Koopmans, L. V. E., et al. 2011, MNRAS, 415, 2215

Barnabè, M. \& Koopmans, L. V. E. 2007, ApJ, 666, 726

Barnabè, M., Spiniello, C., Koopmans, L. V. E., et al. 2013, MNRAS, 436, 253

Barnabè, M., Dutton, A. A., Marshall, P. J., et al. 2012, MNRAS, 423, 1073

Bolton, A. S., Burles, S., Koopmans, L. V. E., et al. 2008, ApJ, 682, 964

Cappellari, M. 2008, MNRAS, 390, 71

Cappellari, M., McDermid, R. M., Alatalo, K., et al. 2012, Nature, 484, 485

一. 2013, MNRAS, 432, 1862

Czoske, O., Barnabè, M., Koopmans, L. V. E., et al. 2008, MNRAS, 384, 987

Genel, S., Vogelsberger, M., Springel, V., et al. 2014, arXiv:1405.3749

Koopmans, L. V. E., Bolton, A., Treu, T., et al. 2009, ApJ, 703, L51

Macciò, A. V., Dutton, A. A., \& van den Bosch, F. C. 2008, MNRAS, 391, 1940

Spiniello, C., Koopmans, L. V. E., Trager, S. C., et al. 2011, MNRAS, 417, 3000

Spiniello, C., Trager, S., Koopmans, L. V. E., \& Conroy, C. 2014, MNRAS, 438, 1483

Thomas, J., Saglia, R. P., Bender, R., et al. 2011, MNRAS, 415, 545

van de Ven, G., Falcón-Barroso, J., McDermid, R. M., et al. 2010, ApJ, 719, 1481

van Dokkum, P. G. \& Conroy, C. 2010, Nature, 468, 940 